\title{
Alcoholic vs non-alcoholic fatty liver in rats: distinct differences in endocytosis and vesicle trafficking despite similar pathology
}

\author{
Karuna Rasineni ${ }^{1,2}$, Daniel D. Penrice ${ }^{1,2}$, Sathish Kumar Natarajan ${ }^{6}$, Mark A. McNiven $^{4}$, Benita L. McVicker ${ }^{1,2}$,
} Kusum K. Kharbanda ${ }^{1,2,3}$, Carol A. Casey ${ }^{1,2,3}$ and Edward N. Harris ${ }^{5^{*}}$

\begin{abstract}
Background: Non-alcoholic and alcoholic fatty liver disease (NAFLD and AFLD, respectively) are major health problems, as patients with either condition can progress to hepatitis, fibrosis, and cirrhosis. Although histologically similar, key differences likely exist in these two models. For example, altered content of several vesicle trafficking proteins have been identified in AFLD, but their content in NAFLD is unknown. In this study, we compared select parameters in NAFLD and AFLD in a rat model.

Methods: We fed either Lieber- DeCarli liquid control or alcohol-containing (35\% as calories) diet (AFLD model) or lean or high-fat (12 or $60 \%$ derived from fat, respectively) pellets (NAFLD model) for 8-10 weeks, $n=8$ in each model. Serum, hepatocytes and liver tissue were analyzed. Liver injury markers were measured in serum, triglyceride content and endocytosis (binding and internalization of ${ }^{125}$ I- asialoorosomucoid) was measured in isolated hepatocytes, and content of selected trafficking proteins (Rab3D, Rab7 and Rab18) were determined in whole liver tissue.

Results: Although liver injury markers and triglyceride content were similar in both models, binding and internalization of ${ }^{125}$ - asialoorosomucoid was significantly impaired in the hepatocytes from AFLD, but not NAFLD, animals. In addition, protein content of the asialoglycoprotein receptor (ASGPR) and three trafficking proteins, Rab3D, Rab7and Rab18, were significantly decreased after alcohol, but not high-fat feeding. Levels of protein carbonylation, amount of glutathione stores, and lipid peroxidation were similar irrespective of the insult to the livers that resulted in fatty liver.
\end{abstract}

Conclusion: Impairments in protein trafficking in AFLD are likely a direct result of alcohol administration, and not a function of fatty liver.

Keywords: Alcoholic fatty liver disease (AFLD), RabGTPase proteins, Asialoglycoprotein receptor (ASGPR), Receptormediated endocytosis, Non-alcohol fatty liver disease (NAFLD)

\section{Background}

Fatty liver disease is a prevalent health risk in modern society, can arise from a variety of etiologies, and can progress to hepatitis, fibrosis, and cirrhosis. A triglyceride content of greater than $5 \%$ in the liver is defined as a fatty liver [1], and there are two primary types: alcoholic fatty liver disease (AFLD) and nonalcoholic fatty liver disease (NAFLD). The first is caused by the consumption of alcohol (ethanol is oxidized by alcohol dehydrogenase to form acetaldehyde

\footnotetext{
* Correspondence: eharris5@unl.edu

${ }^{5}$ Department of Biochemistry, University of Nebraska-Lincoln, Lincoln, NE, USA Full list of author information is available at the end of the article
}

which feeds into the acetyl-CoA pathway and, when in excess, undergoes synthesis of long chain fatty acids) to form triglycerides in hepatocytes [2]. The second is the over-consumption of a high fat, high sugar diet in which excess fat is stored in the liver [3]. NAFLD as a result of a high fat/high sugar diet, also called a Western diet, is very prevalent (20-30\% in US population) and it is projected to be the \#1 causation for liver transplantation in less than 7 years [4]. Indeed, NAFLD afflicts $85-90 \%$ of overweight/ obese individuals of the US [5].

Fatty liver arising from either alcohol consumption or the over-consumption of a high fat/high sugar diet has a 
similar phenotype in that the hepatocytes accumulate triglycerides. Additionally, both are known to result in increased injury markers, such as increased serum alanine aminotransferase (ALT), aspartate aminotransferase (AST) and alkaline phosphatase (ALP). Of interest to our laboratories is that while there is extensive data from a variety of laboratories on the biochemical alterations present in the liver during AFL, little data is present on any of these parameters in NAFLD models. Previous studies have focused on the effects of ethanol exposure which inhibits clathrin-mediated endocytosis by inhibition of plasma membrane fusion [6] and more specifically on the asialoglycoprotein receptor (ASGPR) trafficking and ligand interactions in our lab [7,8]. Additionally, we have studied several small Rab-GTPases known to be involved in vesicle trafficking and show decreased content in livers of AFLD animals [9]. In the present study we asked whether these impairments in ASGPR function and RabGTPase content in AFLD would also occur in the setting of NAFLD.

\section{Methods}

Ethanol was purchased from Pharmaco-AAPER (Brookfield, CT). IRDye infrared secondary antibodies (Abs) and blocking buffer were from Li-COR Biosciences (Lincoln, NE). Protease inhibitor cocktail (P2714-1BTL), phosphatase inhibitor (p0044), collagenase (type IV), human orosomucoid ( $\alpha 1$-acid glycoprotein), and mouse anti-Rab7 antibody were obtained from Sigma (St. Louis, MO). The rabbit polyclonal anti-ASGPR antibody was produced in Dr. Casey's laboratory and has been described previously [10]. Goat anti-Rab 18 antibody was from Santa Cruz Biotechnology (Santa Cruz, CA). Mouse anti-perilipin 2 (anti-PLIN2) antibody was from Fitzgerald (Acton, MA). Mouse anti-actin antibody was obtained from Millipore (Billerica, MA). Rab 3D polyclonal antibody was kindly provided by Mark A. McNiven (Mayo Clinic, Rochester, MN). PureLink RNA Mini Kit and TaqMan Gene Expression Master Mix were purchased from Life Technologies (Grand Island, NY). All other chemicals were obtained from Sigma Chemical Co. unless stated otherwise.

\section{Animals, diet administration, hepatocyte isolation and tissue collection}

All animals received humane care in accordance with the guidelines established by the American Association for the Accreditation of Laboratory Animal Care (AAALAC) and Animal Research: Reporting of In Vivo Experiments (ARRIVE) [11] . All experimental procedures and ethical standards involving animals were reviewed and approved by the Institutional Animal Care and Use Committees at the Veteran's Administration NebraskaWestern Iowa Health Care System Research Service
(IACUC \#11-067-07, approved on Aug. 24, 2014), site of the AFLD studies and University of Nebraska Life Sciences Annex (IACUC \#956, approved on Oct. 23, 2013), site of the NAFLD studies. Male Wistar rats weighing 175 to $200 \mathrm{~g}$ were divided into two groups (AFLD and NAFLD) with 8 pairs in each group to get statistically relevant data (Student's paired $t$ Test). As described previously [12] for the AFLD model, rats were pair-fed with control or EtOH-containing Lieber-DeCarli diets [13] contained $18 \%$ of total calories from protein, $35 \%$ from fat, $11 \%$ from carbohydrate, and $36 \%$ from ethanol. In the control diet, ethanol was replaced isocalorically with maltodextrin. For the NAFLD model, rats were allowed ad libitum access to pellet diet and water. Rats in the HFD group (ResearchDiets \#D08060104) were fed a diet with a caloric formulation of $60 \%$ calories derived from fat (lard; a mixture of mono-, poly- and unsaturated fatty acids), $20 \%$ from carbohydrates (corn starch, maltodextrin), and $20 \%$ from protein (Casein) and rats fed a lean diet (Research Diets \#D12450K with a caloric (kcal) composition of $10.0 \%$ derived from fat (lard), $70 \%$ from carbohydrates (corn starch, maltodextrin) and $20 \%$ from protein (Casein). Both NAFLD and AFLD diets were similar for protein and carbohydrate content with a special care of avoiding sucrose and fructose. These diets have been proven to induce AFLD and NAFLD symptoms and disease that model human alcohol induced and non-alcohol induced fatty liver, respectively. Rats in both alcohol and high fat-diet groups were housed at AAALAC certified institutions (Omaha VAMC and UNL) in approved housing facilities and transported to the laboratories for terminal surgical procedures. Rats in the NAFLD groups were fed ad libitum throughout the study; rats in the AFLD group were fed the ethanol diet ad libitum, and the control rat received an equivalent amount of diet that its pair-fed ethanol consumed. On the day of sacrifice, control animals were meal-fed, to minimize variations in feeding patterns [15]. Rats were sacrificed in the morning hours eight weeks after the initiation of the diet regimen after being anaesthetized with $4 \%$ isoflurane gas mixed with oxygen in a $0.9 \mathrm{~m}^{3}$ chamber. Blood samples were collected via the axillary artery, and serum used for analysis. A piece of whole liver was obtained for histological observation (after knotting a small portion of a small liver lobe with surgical polyester thread); the remaining liver was perfused with isotonic buffers containing collagenase (Type $1 \mathrm{~V}$, Sigma \#C5138) as described previously $[14,15]$.

\section{Clinical chemistry}

The serum profile was measured by a Vetscan chemistry analyzer (Abaxis, Union City, CA). Serum samples were loaded on the Mammalian Liver Profile reagent rotor and read with VetScan VS2 Chemistry Analyzer. The 
Mammalian Liver Profile reagent rotor provided the quantitative measurements of alanine aminotransferase (ALT), alkaline phosphatase (ALP), albumin (ALB), bile acids, and total cholesterol.

\section{Liver histology}

Paraffin-embedded liver tissue sections were processed for hematoxylin/eosin staining and evaluated for steatosis and inflammation.

\section{Immunohistochemistry}

Immunohistochemical staining for lipid droplet protein (PLIN2) was performed as described previously [9]. Briefly, paraffin-embedded liver sections were deparaffinized in xylene and rehydrated in ethanol. Following deparaffinization, slides were subjected to antigen retrieval process with $10 \mathrm{mM}$ sodium citrate buffer ( $\mathrm{pH}$ 6) for $20 \mathrm{~min}$. Sections were incubated overnight with PLIN2 antibody and followed by staining with appropriate Alexa Fluor secondary antibody. Sections were mounted with vectashield mounting medium containing DAPI. Confocal images were acquired using a Zeiss 510META laser scanning confocal microscope.

\section{Triglycerides}

The extraction of lipids from hepatocytes was carried out according to the procedure of Folch and colleagues [16]. Aliquots of lipid extract were saponified to quantify the triglycerides (TGs) using the TG diagnostic kit (Thermo dimethyl adipimidate (DMA) kit; Thermo Electron Clinical Chemistry, Louisville, CO).

\section{Western blot analysis}

Liver homogenate (20 \%) was prepared in $60 \%$ sucrose in TE buffer (10 mM Tris-HCL, 1 mM EDTA, pH 7.4) containing a protease and phosphatase inhibitor cocktail (Sigma, St. Louis, MO). Liver post-nuclear supernatant (PNS) fractions were obtained by centrifugation $(1000 \times$ g) of the homogenate for $10 \mathrm{~min}$. Liver PNS samples were separated by $12 \%$ SDS-PAGE, blotted on nitrocellulose and proteins were detected with appropriate primary antibodies and then immunoreactive proteins were visualized and quantified using the Odyssey Infrared Imager and associated software.

\section{Preparation and labeling of asialoorosomucoid (ASOR)} Human orosomucoid (Sigma, St. Louis, MO) was desialylated by the neuraminidase procedure as described by Oka and Weigel [17]. ${ }^{125}$ I-ASOR was prepared by the procedure described previously [15]. Briefly, $125 \mu \mathrm{g}$ of ASOR in $200 \mu \mathrm{l}$ of PBS was reacted with $38 \mu \mathrm{g}$ 1,3,4,6-tetrachloro$3 \alpha, 6 \alpha$-diphenyl-glycoluril dried oxidizing reagent coated on the bottom of a glass tube and $0.3 \mathrm{mCi} \mathrm{Na}{ }^{125} \mathrm{I}$ at room temperature for $15 \mathrm{~min}$. The labeled ASOR was then separated from unincorporated $\mathrm{Na}^{125} \mathrm{I}$ with a PD-10 column and quantified by the Bradford assay.

\section{Measurements of ASOR binding and internalization}

Assessment of internalization of ASOR was determined by a modification of our previous studies [15]. Briefly, hepatocytes isolated from AFLD and NAFLD animals were plated in triplicate on collagen coated 24-well plates at a concentration of $10^{5}$ cells/well and incubated at $37{ }^{\circ} \mathrm{C}$ with $1.0 \mu \mathrm{g} / \mathrm{mL}{ }^{125} \mathrm{I}$-ASOR $(25 \mathrm{nM})$ for various periods of time $(0,30,60,90 \& 120 \mathrm{~min})$. Receptor specificity was measured in replicate wells containing excess unlabeled ASOR to assess background levels. After each incubation period, cells were washed with phosphatebuffered saline and the amount of radioactive ligand in cell lysates was determined and normalized to total cell lysate protein.

\section{mRNA analysis /Analysis of gene expression}

For quantification of mRNA, RNA was isolated from liver pieces using a PureLink RNA Mini Kit (Invitrogen, Carlsbad, CA) and was reverse transcribed from $1 \mu \mathrm{g}$ of total RNA using oligo-dT primers and the Transkriptor kit (Roche Applied Science). To determine gene expression levels, real-time PCR reactions were performed using rat-specific primers from the TaqMan Gene Expression Assay System (Rab3D; catalog \# rn00756153; Rab 7, catalog \# rn00592246; Rab18, catalog \# rn01526466; ASGPR, catalog \# rn00560750) and samples were analyzed in the 7500 Real Time PCR System (Applied Biosystems, Carlsbad, CA). The $\Delta \Delta \mathrm{Ct}$ method was used to determine the fold change using actin for normalization.

\section{Serum hormone quantification}

Metabolic hormones; leptin, amylin and insulin were measured using the Multiplex MAP Magnetic Beadbased immunoassay kits (Millipore Corp. Billerica, MA). The assay was conducted according to the manufacturer's instructions using handheld magnetic separator block for 96-well flat bottom plates (Millipore, Millipore Corp) and analyzed using the Luminex 200 system (Luminex Corp., Austin, TX). All samples were run in duplicate and standards supplied by the manufacturer were run on each plate. Mean fluorescence intensity was analyzed using the BioPlex manager software version 5.0 (Bio-Rad, Hercules, CA) and compared to a standard curve to calculate the concentrations. Values below the range of the standard curve were set to the lower limit of detection.

Selected oxidative stress-related parameters in liver tissue Pro-oxidant formation was assessed by measuring protein carbonyl content as described [18] using 2, 4-dinitrophenylhydrazine and calculated using an extinction 
coefficient of $22 \mathrm{mM}^{-1} \mathrm{~cm}^{-1}$. In addition, the extent of lipid peroxidation was assessed by quantitating thiobarbituric acid reactive substances (TBARS) following the procedure of Uchiyama \& Mihara [19] using malondialdehyde (MDA) as a standard. Anti-oxidant defense was quantified by measuring total and oxidized glutathione (GSSG) using the enzymatic method [20].

\section{Statistical analysis}

The results are expressed as mean $\pm \mathrm{SEM}$. Comparison between control and their respective experimental animals was analyzed using the Student's $t$-test. p-values of $<0.05$ were considered significant.

\section{Results}

Morphology of liver tissue in alcohol and non-alcohol induced fatty liver

At the outset of these experimental procedures, we confirmed those rats on the Lieber-DeCarli $(\mathrm{EtOH})$ diet and their cohorts on the high fat diet (HFD) had fatty liver by H\&E staining of the liver tissue (Fig. 1). The increased fat content was also quantitated by measuring triglyceride content in purified hepatocytes. The amount of triglyceride was similar between the control groups for both AFLD and NAFLD animals, and in the alcoholfed and high-fat groups, indicating that induction of fatty liver by both diets were equivalent (Fig. 1e). PLIN 2
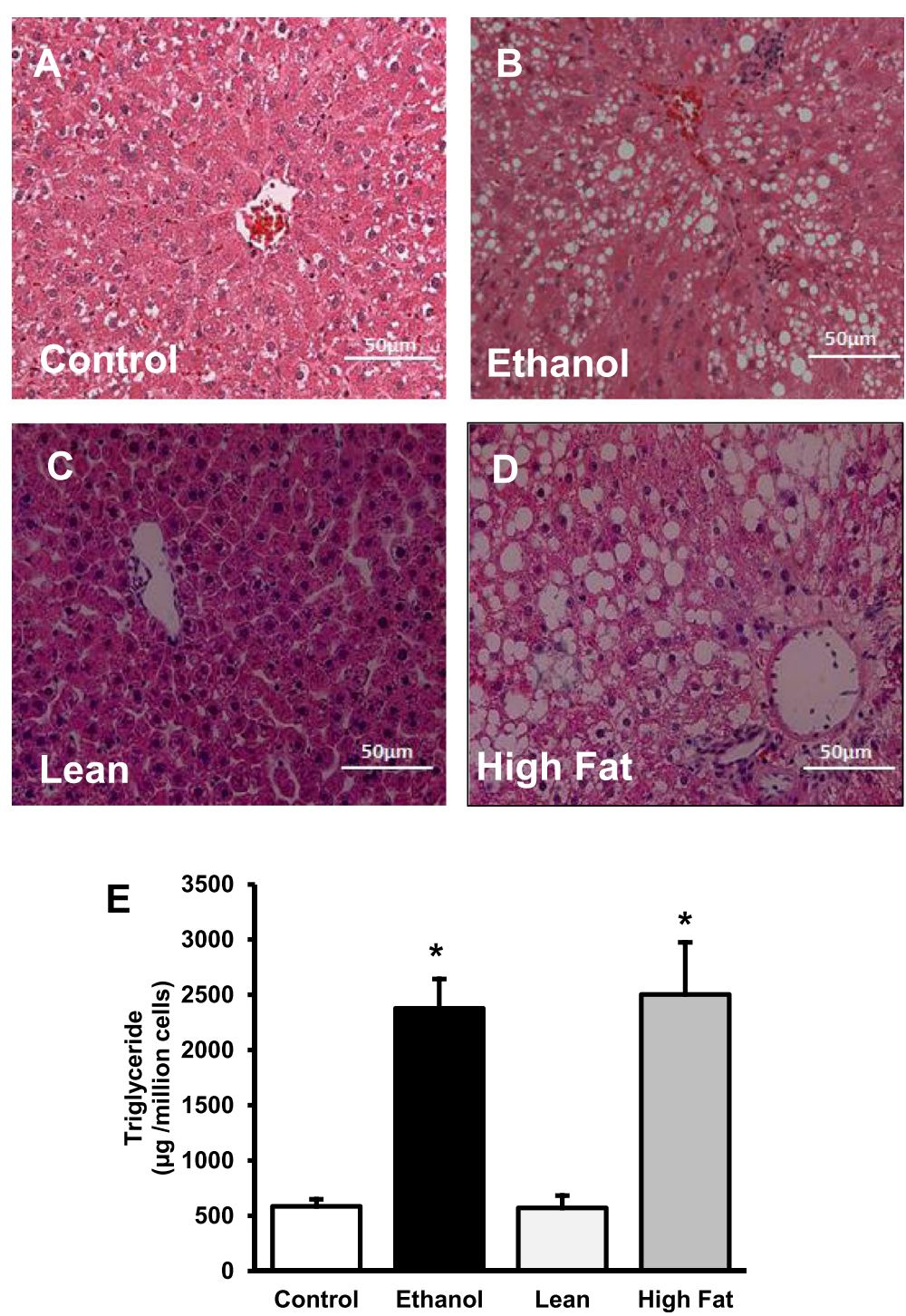

Fig. 1 Triglyceride accumulation in the hepatocytes of control and EtOH-fed rats (a, b) and of lean and HFD rats (c, d). Hematoxylin and Eosin staining was performed on paraffin sections. Images are representative of each pair-fed group of $n=8$. Magnification, 200x. e Quantification of lipid content in isolated hepatocytes. Hepatocyte TGs were extracted with chloroform: methanol (2:1) and amount of glycerol were measured in each sample. TG levels were significantly increased in both AFLD and NAFLD rats when compared to their respective controls. Values are means $\pm \mathrm{SEM}, n=8,{ }^{*} p \leq 0.05$ 
staining (a lipid droplet marker) also revealed increases in LD accumulation between controls and experimental groups (Fig. 2).

\section{Comparative serum profiles in NAFLD and AFLD}

Serum from each animal was tested for markers of liver damage using the Liver Profile Rotorary disc (Abaxis). We measured alkaline phosphatase (ALP) is a marker for a number of disorders including blocked bile ducts [21] and some forms of cancer [22], but in our models, it is a sign of cholangiocyte stress and/or damage. ALP levels in the experimental animals (EtOH-fed or HFD) were significantly higher than the matched controls (Fig. 3a). Alanine aminotransferase (ALT) is an indicator of hepatocyte cell death [23] was also increased in both the high-fat groups (Fig. 4b). Bile acids (Fig. 3c) and cholesterol (Fig. 3d) were significantly higher in the $\mathrm{EtOH}$-fed rats, but no significant differences were identified between high-fat and lean controls in NAFLD model. These results suggest that the synthesis of lipids from alcohol metabolic precursors has a greater impact on cholesterol biosynthesis than the absorption of fatty acids and esterification of triglycerides [24, 25]. Levels of albumin, an abundant serum protein produced by hepatocytes, were not affected in either model (Fig. 3e).

We also analyzed pancreatic hormones level in serum using the Rat Metabolic Hormone Panel provided by Milliplex systems. Insulin resistance has been shown to be associated with NAFLD/AFLD [26-28] and, not surprisingly, our NAFLD model demonstrated hyperinsulinemia (Fig. 4a). In contrast, despite similar glucose levels observed in both control and ethanol rats in the fed condition (data not shown); insulin levels in the serum of EtOH-fed rats were lower than the controls. In NAFLD animals, along with increased insulin in the serum (Fig 4a), we also observed increased glucose (data not shown). These values were obtained in fed animals, and further studies on insulin resistance and determination of HOMA-IR will be important to examine in fasted animals. Like insulin, amylin is a peptide hormone produced by pancreatic beta cells and is co-secreted with insulin to decrease gastric emptying and increase satiety. Levels of amylin were unchanged in HF-fed rats suggesting that pancreatic beta cells are normal. In AFLD rats, the levels were significantly decreased in the EtOH-fed rats compared to the dietary control (Fig. 4b). The satiety hormone, leptin, was increased in NAFLD rats and unchanged in AFLD rats (Fig. 4c). These results may be reflective of the administration of the diet which is ad libitum for NAFLD and calorie restricted for the AFLD diet or the effects of ingested alcohol on pancreatic function.

\section{Receptor-mediated endocytosis in AFLD versus NAFLD}

Next, we focused on hepatocyte endocytosis in the isolated primary cells from the two models. Our previous work has shown that alcohol impairs multiple aspects of the process of receptor-mediated endocytosis (RME), using the ASGPR as a model $(8-11,15)$. In the present
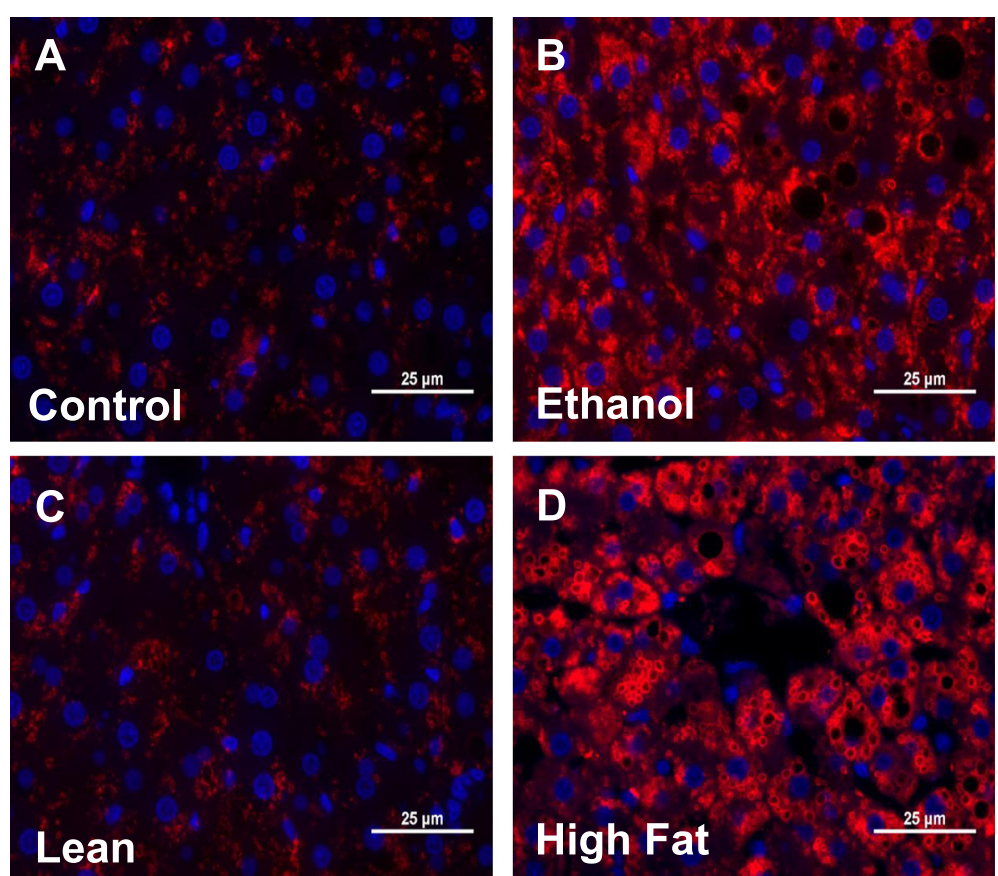

Fig. 2 PLIN2 staining for lipid droplets: Liver sections from Control and EtOH-fed $(\mathbf{a}, \mathbf{b})$ and Lean and HFD(c, $\mathbf{d})$ rats both show increased lipid droplet accumulation (as identified by PLIN2) when compared to their controls. Images are representative of each pair-fed group of $n=8$. Magnification, 400x 

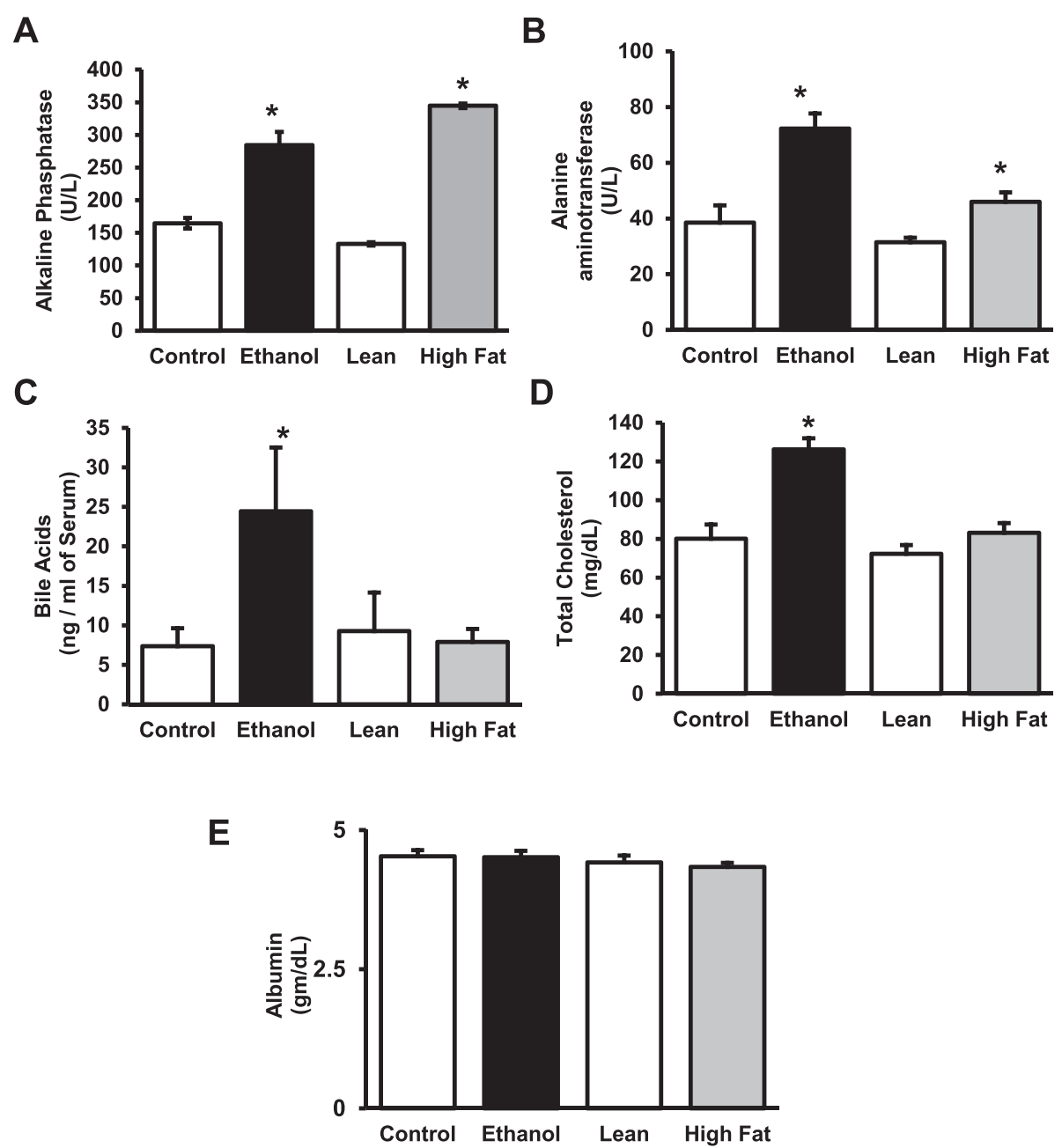

Fig. 3 Liver markers in serum of AFLD/NAFLD rats. The VetScan Liver Profile system for analyzing serum revealed specific changes in enzyme and molecular markers as indicated. The EtOH-fed and HFD cohorts were compared against their respective dietary controls. Values are means \pm SEM, $n=8,{ }^{*} p \leq 0.05$

studies, we used a radiolabeled ligand $\left({ }^{125} 1\right.$-Acid glycoprotein or orosomucoid (ASOR)), we found that ASGPR internalization rates with cargo in AFLD are $50 \%$ of the control (Fig. 5a), in contrast to NAFLD model in which endocytosis rates are unchanged between control and HF-fed rats. Follow-up binding studies at $4{ }^{\circ} \mathrm{C}$, indicate that the level of surface receptors is lower in AFLD hepatocytes (Fig. 5b), but not in NAFLD hepatocytes. ASGPR protein and mRNA levels in the AFLD rats were decreased by about 30 and $45 \%$, respectively. In NAFLD rats, ASGPR levels were slightly increased as compared with the control rats, but these differences were not statistically distinguishable (Fig. 5c \& d).

\section{Vesicle protein trafficking defects in AFLD versus NAFLD}

In previous studies, we have identified altered protein content for several Rab GTPases in the alcoholic fatty liver [9]. In particular, we identified decreased content for Rab3D (involved in exocytosis), Rab7 (involved in endocytosis/autophagy) and Rab18 (involved in Golgi-endoplasmic reticulum transfer). In the current study, we measured content of these proteins in livers of AFLD and NAFLD animals (Fig. 6a). Similar to what we have previously identified, ethanol treatment significantly decreased the content of Rab3D (75 \%), Rab7 (25 \%) and Rab18 (16 \%) in liver (Fig. 6b). mRNA for Rab3D, Rab7 and Rab18 were also significantly impaired in the alcohol-fed, but not the high fat, non-alcoholic animals (Fig. 6c). In contrast, HFD animals in the NAFLD model did not show any significant change in Rab7 and Rab18, and increased Rab3D content. The mRNA profile for the NAFLD animals reflected the protein expression (Fig. 6c), and was unchanged.

\section{Oxidant stress and anti-oxidant defenses}

Oxidative stress and lipotoxicity are known to occur in NAFLD [29] and AFLD [30]. In regards to the oxidative 
A

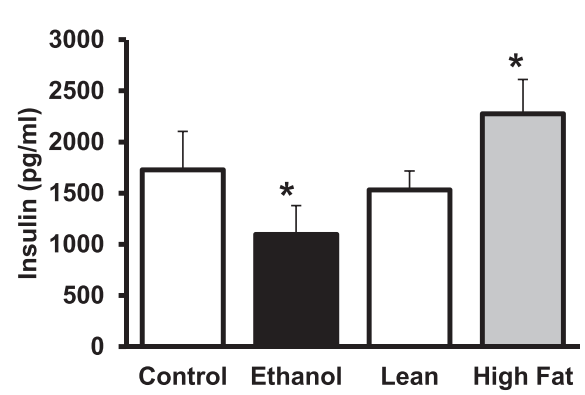

C

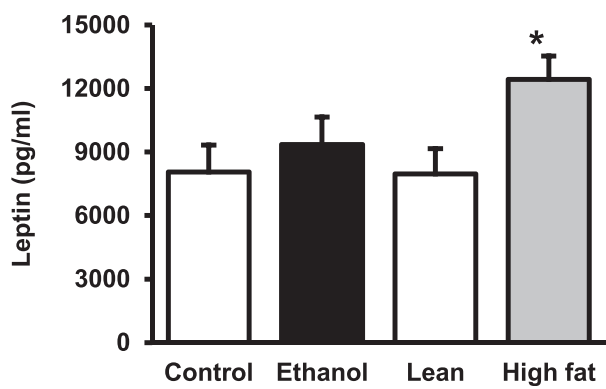

B

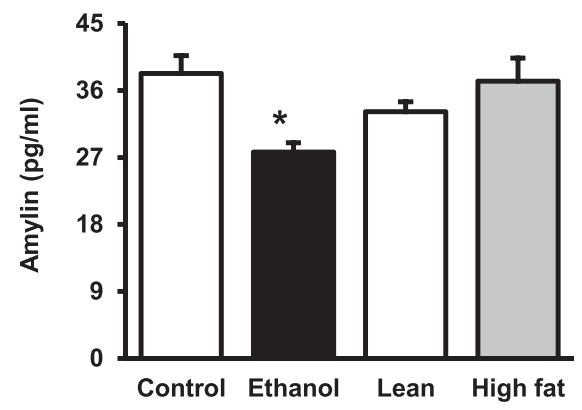
Panel (Millipore), and compared against their respective dietary controls. Values are means \pm SEM, $n=8,{ }^{*} p \leq 0.05$

stress in both comparative model systems, we observed a significant increase in protein carbonyls content, TBARS (a by-product of lipid peroxidation) and oxidized glutathione (GSSG) in livers of both ethanol-fed and high-fed animals compared to their respective controlfed animals (Fig. 7a-d). In contrast, $\sim 25 \%$ lower content of reduced glutathione (GSH) in livers of both EtOH-fed and HFD rats compared to their controls was observed.

\section{Discussion}

One common classical effect of both chronic alcohol intake and high-fat diet consumption is the presence of a fatty liver, with similar histology (essentially indistinguishable) between the two types. With the increased incidence of NAFLD in the general population, it will be important to elucidate differences in mechanisms of fatty liver pathogenesis between fatty liver induced by alcohol administration (AFLD) as compared to that observed under nutrient-induced high-fat diet conditions. In this article, we have demonstrated the characteristic differences of AFLD and NAFLD regarding selected metabolic parameters and important aspects of membrane trafficking by focusing on some well-identified defects which have been involved in AFLD, and compared these results with a model of diet-induced fatty liver.

For studies reported here, Wistar rats were administered either alcohol (as $36 \%$ of calories as ethanol, $35 \%$ as fat, $11 \%$ carbohydrate) in a nutritionally adequate liquid diet or a high fat diet $(60 \%$ of calories from fat, $20 \%$ calories from carbohydrate) for 8 weeks. Thus, the composition of diet and the calorie intake are different, but both are well accepted models to induce AFLD and NAFLD respectively. Moreover, it has been shown that feeding alcohol (35\%) containing or high fat-fed diets led to histopathological hepatic changes similar to human AFLD and NAFLD [31, 32]. The mechanisms proposed to play a role in the development of alcoholic fatty liver involve an increase in the fatty acid synthesis, decrease in fatty acid oxidation and impaired VLDL secretion [33-36]. In high fat-fed conditions, fatty liver is believed to follow from the development of insulin resistance, an imbalance between hepatic lipid intake, synthesis, degradation and secretion [37, 38]. Despite the differences in underlying mechanisms, the pathological features are the same in both conditions. Thus, we took an interesting approach to elucidate the metabolic differences and similarities between these diseases. As expected, we observed increased hepatic lipid deposition in both models and the degree and distribution of lipid droplets were similar. Further assessment of hepatic function through the measure of serum enzymes and metabolites revealed both models showed significantly higher ALT and ALP when compared to their respective controls. These increases are expected indicators of liver damage. AST levels were not measured with the VetScan chemistry rotor and are not a reliable marker in simple 


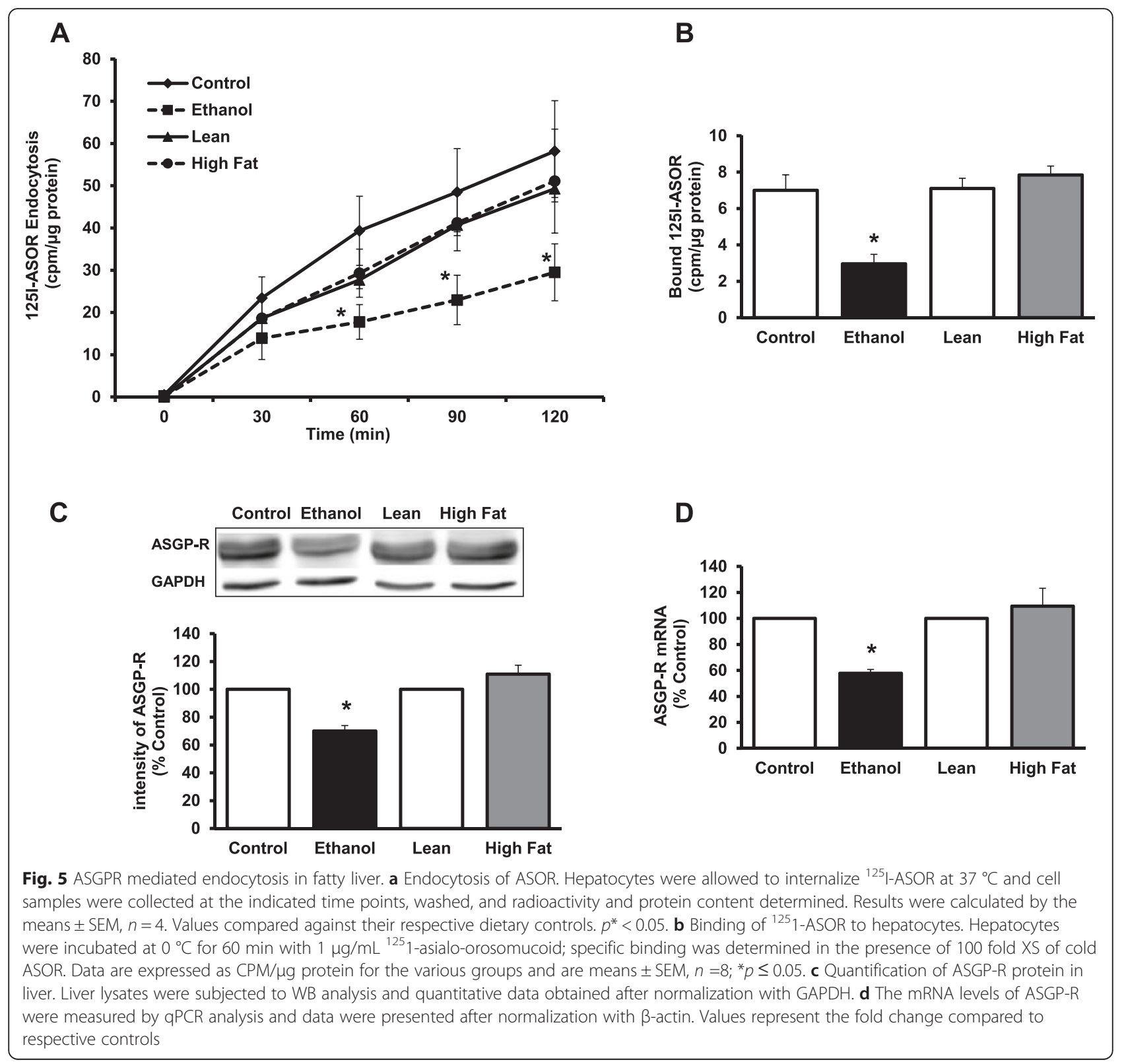

steatohepatitis [39]. Serum bile acids were significantly increased in the ethanol-fed AFLD, but not the high-fat NAFLD animals when compared to their respective controls. An increase in serum bile acids for the AFLD rats suggest an intrahepatic cholestasis, similar to what has been reported for serum profiles of ASH and NASH patients [40-42].

Extra hepatic hormone factors are also known to critically modulate hepatic lipid metabolism. In this study, we measured insulin, amylin and leptin, all of which are known to participate in the complex process of energy homeostasis. In ethanol-fed animals, the pancreatic hormones, insulin and amylin, were found to be significantly decreased compared to the pair-fed controls. Levels of the adipocyte hormone, leptin, were not altered in sera of EtOH-fed animals. The decreased plasma levels of pancreatic hormones (insulin and amylin) likely indicate impaired pancreatic function in the setting of AFLD. In contrast, NAFLD rats fed with the HFD showed hyperinsulinemia and increased leptin levels compared to the lean controls. Amylin levels were unchanged between experimental and control animals of the NAFLD group. Hyperinsulinemia and insulin resistance are common in both diabetes and obesity, and in animal models, Samuel et al. reported that the ability of insulin to suppress hepatic glucose production is diminished in rats feeding with HFD 


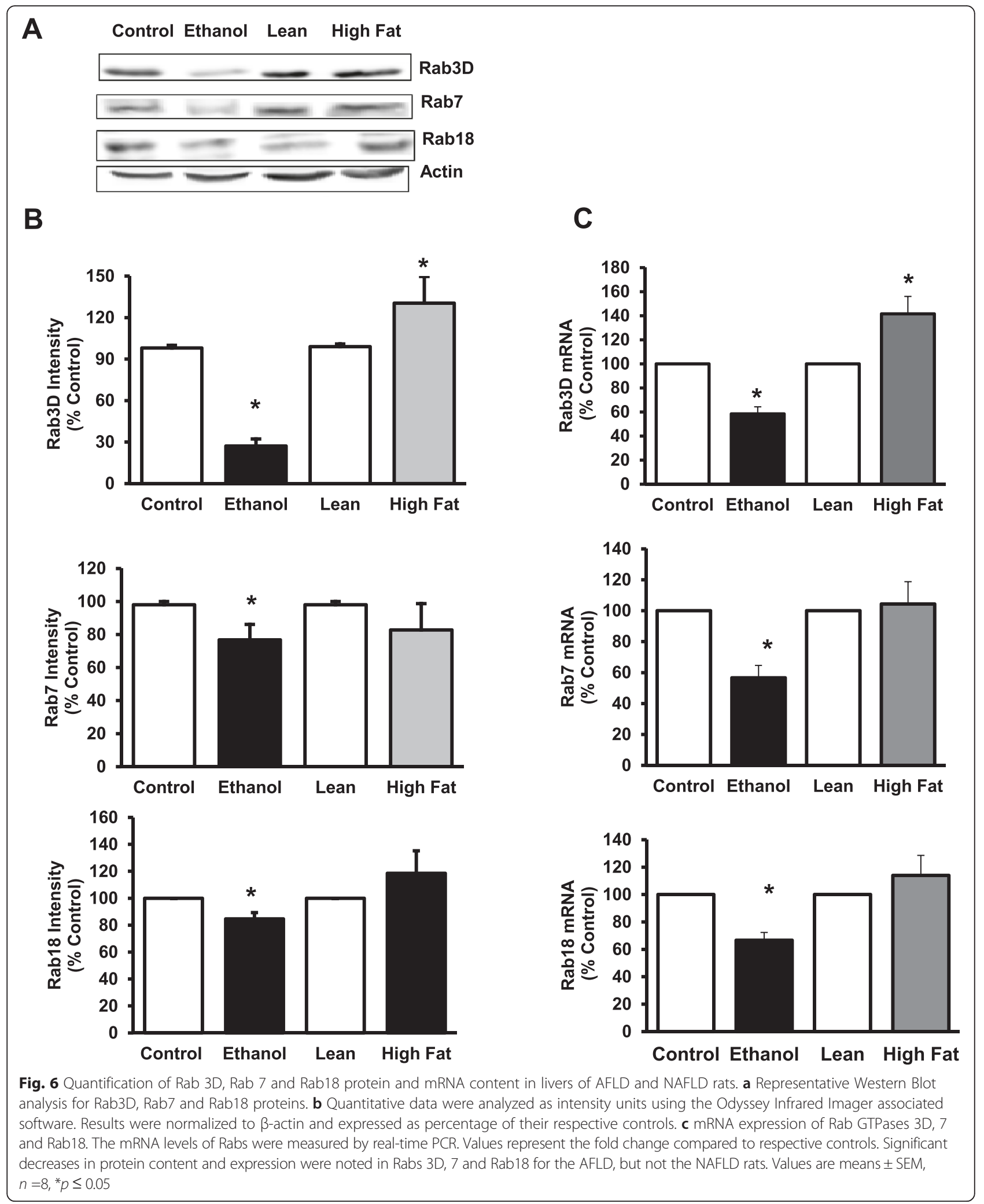

for 3 days [43]. Thus, impaired insulin function could lead to compensatory hyperinsulinemia, where the body attempts to balance the reduced effect of insulin by producing and releasing more insulin. Along with hyperinsulinemia, we also observed increased leptin levels in NAFLD, which is likely due to leptin resistance $[44,45]$. 


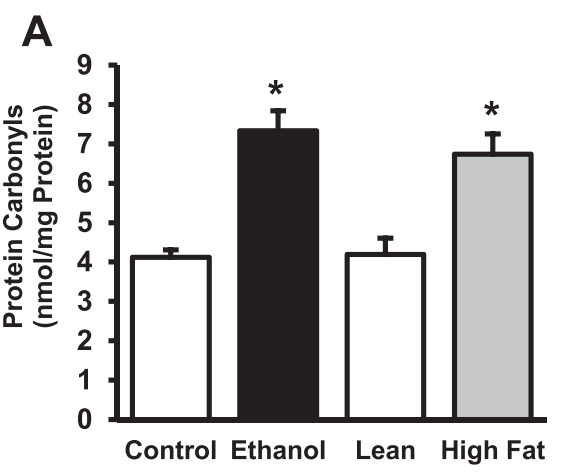

\section{B}

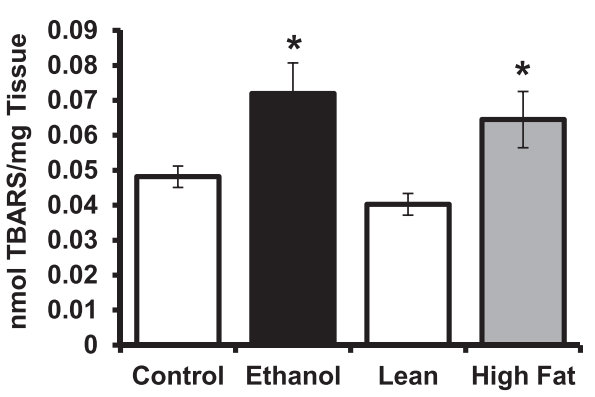

C

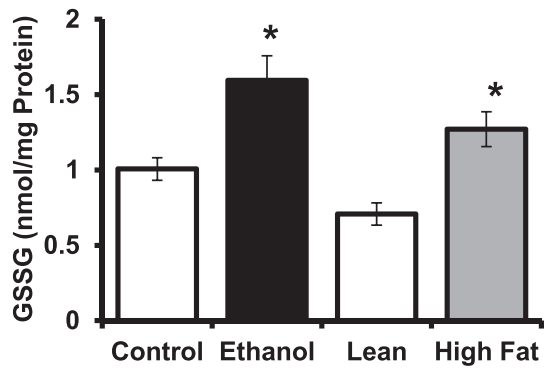

D

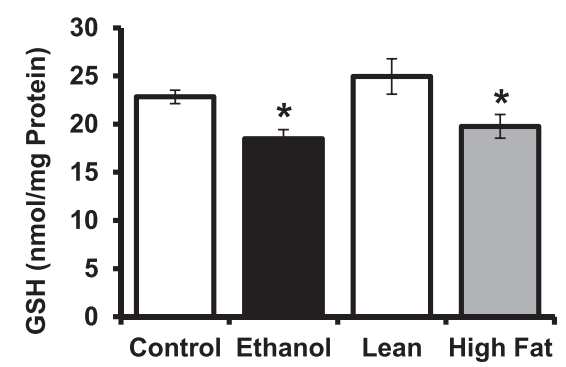

Fig. 7 Quantification of selected parameters of oxidative stress and anti-oxidant defenses in livers of AFLD and NAFLD rats. a Protein carbonyls; (b) TBARS; (c) Oxidized GSH (GSSG) (d) Reduced GSH levels were determined as indicated in the Methods section. Values are means \pm SEM, $n=8$, * $p \leq 0.05$ (compared to their respective controls)

The mechanisms for progression of alcoholic liver diseases are likely multifactorial and include multiple mechanisms, with impaired protein trafficking as one likely mechanism. Dr. Casey and her laboratory have studied the events of impaired RME and its consequences in progression of liver disease by using the hepatocyte specific ASGPR. Chronic ethanol administration has been shown to alter multiple aspects of the hepatic RME pathway, including decreases in receptor expression, protein content, ligand binding, ligand internalization and receptor recycling $[10,15,46,47]$. These impairments were identified as early as one week of ethanol administration, and were most prominent in hepatocytes isolated from the centrilobular region of the liver [48]. Consistent with those previous studies, we report here that ethanol administration showed significant impairments of ${ }^{125}$ I-ASOR ligand binding and internalization in hepatocytes from EtOH-fed animals when compared to controls. In parallel to decreased binding, we also observed decreases in ASGPR expression and its content in ethanol-fed rats. In contrast, no difference in binding or internalization was found in the hepatocytes isolated from the lean and high-fat animals, indicating specific alcohol-related impairments to endocytosis by the ASGPR.

In addition to endocytosis by the ASGPR, we have recently reported that ethanol administration results in decreased protein levels of several small GTPases known to play an essential role in controlling membrane trafficking of endo- and exocytic pathways [9]. Importantly, we showed that protein content of three Rabs known to be involved in vesicle trafficking, endocytosis and Golgiendoplasmic reticulum transport (Rab3D, Rab7 and Rab 18) were are decreased in after alcohol administration, while others (Rab 1, 2 and 5) were unaffected. In the present study we had a particular interest in the Rabs which were sensitive to alcohol administration, and here, we show that in the AFLD animals, there was a marked, $75 \%$ decrease in the content of Rab3D GTPase in liver, along with a significant decrease in Rab3D mRNA. Similarly, protein and mRNA content of Rab7 were also significantly lower in ethanol-fed, but not HFD animals, and Rab18 content and mRNA showed similar results. None of these proteins showed significant differences between the NAFLD groups. All of these Rabs could play a direct role ASGPR trafficking, which was impaired in AFLD, but not NAFLD rats. Since increased fat in the form of lipid droplets in AFLD hepatocytes arise from ethanol and acetaldehyde metabolism, it is possible that these key metabolites play a role in altered Rab function. The appearance of fatty liver in AFLD is in contrast to NAFLD hepatocytes which accumulate lipid due to mass action of bulk lipid availability. In simple steatosis without inflammation, which is portrayed by our NAFLD 
animal model, the liver is still relatively healthy and just beginning to show injury as measured with the ALP and ALT levels. In this state, the liver may serve as a secondary site for lipid storage due to the metabolism of high concentrations of free fatty acids absorbed by the small intestine, packaged into chylomicrons, and delivered in the lymphatics and blood stream and, ultimately, to the liver. The trafficking machinery in hepatocytes is still functional as evidenced by the normal levels of Rabs and ASGPR activity. We hypothesize that vesicle trafficking in and out of the cell is normal in NAFLD, but liposome autophagy and trafficking is impaired due to the toxic effects of ethanol metabolism to the cellular machinery in AFLD. Follow-up experimentation examining lipiddroplet trafficking in the NAFLD and how that is compared with what is known in AFLD is currently under investigation.

These findings suggest that even though both AFLD and NAFLD show the same characteristics of fat accumulation and selected examined parameters of liver pathology (including increased oxidative stress in both AFLD and NAFLD models), the impairments in membrane trafficking in the ethanol fed animals is likely a result of alcohol administration, and not from these observed changes in high-fat or EtOH-fed rats. In EtOH-fed conditions, ethanol metabolism, in addition to promoting fatty acid synthesis, also generates highly reactive acetaldehyde and reactive oxygen species (ROS) by alcohol dehydrogenase (ADH) and CYP2E1 (cytochrome P450, family 2, subfamily E, polypeptide 1) [49]. Since we observed similar changes in the selected parameters of oxidant stress and anti-oxidant defenses irrespective of the insult to the livers that resulted in fatty liver, it is likely that the observed differences we have identified are related to metabolites of alcohol. Indeed, previous work has shown in cultured cells that when alcohol metabolism is blocked by the addition of pyrazole, impaired endocytosis by the hepatic ASGPR required ethanol oxidation [50]. Additionally, our previous work showed that in the presence of an inhibitor of aldehyde dehydrogenase (cyanamide), that the impairments to endocytosis were attenuated [51].

\section{Conclusion}

We provide evidence that the observed endocytosis and vesicle protein content in AFLD animals are most likely effects of ethanol metabolism in the liver which is not seen in NAFLD.

\section{Abbreviations}

AFLD: alcoholic fatty liver disease; ALP: alkaline phosphatase; ALT: alanine aminotransferase; ASGPR: asialoglycoprotein receptor;

ASOR: asiaoloorosomucoid; AST: aspartate aminotransferase; EtOH: ethanol; GSH: reduced glutathione; GSSG: oxidized glutathione; HFD: high fat diet: LD: lipid droplet; MDA: malondialdehyde; NAFLD: non-alcoholic fatty liver disease; RME: receptor mediated endocytosis; TBARS: thiobarbituric acid reactive substances.

\section{Competing interests}

The author(s) declare that they have no competing interests.

\section{Authors' contributions}

$K R$, DP, SKN, BLM and KKK carried out the experimental work of the study. MAM provided intellectual contributions and some study design. CAC and ENH conceived the study and drafted the manuscript. All authors read and approved the final manuscript.

\section{Acknowledgements}

This work was supported by the Nebraska Research Initiative (NRI) to E.N.H and C.A.C., NIH grant 5R01AA020735 to C.A.C. and M.A.M., and NIH grant 5R01HL094463 to ENH.

\section{Author details}

${ }^{1}$ Department of Internal Medicine, University of Nebraska Medical Center, Omaha, NE, USA. ${ }^{2}$ The Liver Study Unit, VA Nebraska-Western lowa Health Care System, Omaha, NE, USA. ${ }^{3}$ Department of Biochemistry and Molecular Biology, University of Nebraska Medical Center, Omaha, NE, USA.

${ }^{4}$ Department of Biochemistry and Molecular Biology and the Center for Digestive Diseases, Mayo Clinic, Rochester, MN, USA. ${ }^{5}$ Department of Biochemistry, University of Nebraska-Lincoln, Lincoln, NE, USA. ${ }^{6}$ Dept. of Nutrition and Health Sciences, University of Nebraska-Lincoln, Lincoln, NE, USA.

Received: 22 October 2015 Accepted: 12 February 2016

Published online: 29 February 2016

\section{References}

1. Angulo P, Lindor KD. Non-alcoholic fatty liver disease. J Gastroenterol Hepatol. 2002;17(Suppl):S186-90.

2. Lieber CS. Hepatic, metabolic and toxic effects of ethanol: 1991 update. Alcohol Clin Exp Res. 1991;15(4):573-92.

3. Musso G, Gambino R, Cassader M. Recent insights into hepatic lipid metabolism in non-alcoholic fatty liver disease (NAFLD). Prog Lipid Res. 2009;48(1):1-26.

4. Heimbach J. Debate: A bridge too far-liver transplantation for nonalcoholic steatohepatitis will overwhelm the organ supply. Liver Transpl. 2014;20 Suppl 2:S32-7.

5. Chalasani N, Younossi Z, Lavine JE, Diehl AM, Brunt EM, Cusi K, et al. The diagnosis and management of non-alcoholic fatty liver disease: practice Guideline by the American Association for the Study of Liver Diseases, American College of Gastroenterology, and the American Gastroenterological Association. Hepatology. 2012;55(6):2005-23.

6. Fernandez DJ, McVicker BL, Tuma DJ, Tuma PL. Ethanol selectively impairs clathrin-mediated internalization in polarized hepatic cells. Biochem Pharmacol. 2009;78(6):648-55.

7. McVicker BL, Casey CA. Effects of ethanol on receptor-mediated endocytosis in the liver. Alcohol. 1999;19(3):255-60.

8. McVicker BL, Tuma DJ, Kubik JA, Hindemith AM, Baldwin CR, Casey CA. The effect of ethanol on asialoglycoprotein receptor-mediated phagocytosis of apoptotic cells by rat hepatocytes. Hepatology. 2002;36(6):1478-87.

9. Rasineni K, McVicker BL, Tuma DJ, McNiven MA, Casey CA. Rab GTPases associate with isolated lipid droplets (LDs) and show altered content after ethanol administration: potential role in alcohol-impaired LD metabolism. Alcohol Clin Exp Res. 2014:38(2):327-35.

10. Tworek BL, Tuma DJ, Casey CA. Decreased binding of asialoglycoproteins to hepatocytes from ethanol-fed rats. Consequence of both impaired synthesis and inactivation of the asialoglycoprotein receptor. J Biol Chem. 1996; 271(5):2531-8.

11. Kilkenny C, Browne WJ, Cuthill IC, Emerson M, Altman DG. Improving bioscience research reporting: the ARRIVE guidelines for reporting animal research. PLoS Biol. 2010;8(6):e1000412.

12. Casey CA, McVicker BL, Donohue Jr TM, McFarland MA, Wiegert RL, Nanji AA. Liver asialoglycoprotein receptor levels correlate with severity of alcoholic liver damage in rats. J Appl Physiol. 2004;96(1):76-80.

13. Lieber CS, DeCarli LM. Liquid diet technique of ethanol administration: 1989 update. Alcohol Alcohol. 1989;24(3):197-211. 
14. Gopalakrishnan S, Harris EN. In vivo liver endocytosis followed by purification of liver cells by liver perfusion. J Vis Exp. 2011;10(57):3138.

15. Casey CA, Kragskow SL, Sorrell MF, Tuma DJ. Chronic ethanol administration impairs the binding and endocytosis of asialo-orosomucoid in isolated hepatocytes. J Biol Chem. 1987;262(6):2704-10.

16. Folch J, Lees M. Sloane Stanley GH. A simple method for the isolation and purification of total lipides from animal tissues. J Biol Chem. 1957;226(1):497-509.

17. Oka JA, Weigel PH. Recycling of the asialoglycoprotein receptor in isolated rat hepatocytes. Dissociation of internalized ligand from receptor occurs in two kinetically and thermally distinguishable compartments. J Biol Chem. 1983;258(17):10253-62

18. Natarajan SK, Thangaraj KR, Eapen CE, Ramachandran A, Mukhopadhya A, Mathai $M$, et al. Liver injury in acute fatty liver of pregnancy: possible link to placental mitochondrial dysfunction and oxidative stress. Hepatology. 2010; 51(1):191-200

19. Mihara M, Uchiyama M. Determination of malonaldehyde precursor in tissues by thiobarbituric acid test. Anal Biochem. 1978;86(1):271-8.

20. Tietze F. Enzymic method for quantitative determination of nanogram amounts of total and oxidized glutathione: applications to mammalian blood and other tissues. Anal Biochem. 1969;27(3):502-22.

21. Chaudhary A, Dhar P, Tomey S, Sachdev A, Agarwal A. Segment III cholangiojejunostomy for carcinoma of the gallbladder. World J Surg. 1997; 21(8):866-70. discussion 870-861.

22. Li-Fern H, Rajasoorya C. The elevated serum alkaline phosphatase-the chase that led to two endocrinopathies and one possible unifying diagnosis. Eur J Endocrinol. 1999;140(2):143-7.

23. Canbakan B, Senturk H, Canbakan M, Toptas T, Tabak O, Balci H, et al. Is alanine aminotransferase level a surrogate biomarker of hepatic apoptosis in nonalcoholic fatty liver disease? Biomark Med. 2010;4(2):205-14.

24. Duryee MJ, Willis MS, Schaffert CS, Reidelberger RD, Dusad A, Anderson DR, et al. Precision-cut liver slices from diet-induced obese rats exposed to ethanol are susceptible to oxidative stress and increased fatty acid synthesis. Am J Physiol Gastrointest Liver Physiol. 2014;306(3):G208-17.

25. Duarte JA, Carvalho F, Pearson M, Horton JD, Browning JD, Jones JG, et al. A high-fat diet suppresses de novo lipogenesis and desaturation but not elongation and triglyceride synthesis in mice. J Lipid Res. 2014;55(12):2541-53.

26. Marchesini G, Brizi M, Morselli-Labate AM, Bianchi G, Bugianesi E, McCullough AJ, et al. Association of nonalcoholic fatty liver disease with insulin resistance. Am J Med. 1999;107(5):450-5.

27. Adiels M, Westerbacka J, Soro-Paavonen A, Hakkinen AM, Vehkavaara S, Caslake MJ, et al. Acute suppression of VLDL1 secretion rate by insulin is associated with hepatic fat content and insulin resistance. Diabetologia. 2007:50(11):2356-65.

28. Ramirez T, Longato L, Dostalek M, Tong M, Wands JR, de la Monte SM. Insulin resistance, ceramide accumulation and endoplasmic reticulum stress in experimental chronic alcohol-induced steatohepatitis. Alcohol Alcohol. 2013:48(1):39-52.

29. Paradies G, Paradies V, Ruggiero FM, Petrosillo G. Oxidative stress, cardiolipin and mitochondrial dysfunction in nonalcoholic fatty liver disease. World J Gastroenterol. 2014;20(39):14205-18.

30. Song BJ, Akbar M, Abdelmegeed MA, Byun K, Lee B, Yoon SK, et al. Mitochondrial dysfunction and tissue injury by alcohol, high fat, nonalcoholic substances and pathological conditions through posttranslational protein modifications. Redox Biol. 2014;3:109-23.

31. Svegliati-Baroni G, Candelaresi C, Saccomanno S, Ferretti G, Bachetti T, Marzioni $\mathrm{M}$, et al. A model of insulin resistance and nonalcoholic steatohepatitis in rats: role of peroxisome proliferator-activated receptoralpha and n-3 polyunsaturated fatty acid treatment on liver injury. Am J Pathol. 2006;169(3):846-60

32. Li Y, Hai J, Li L, Chen X, Peng H, Cao M, et al. Administration of ghrelin improves inflammation, oxidative stress, and apoptosis during and after non-alcoholic fatty liver disease development. Endocrine. 2013;43(2):376-86.

33. Grunnet N, Kondrup J. The effect of ethanol on the beta-oxidation of fatty acids. Alcohol Clin Exp Res. 1986;10(6 Suppl):64S-8.

34. Venkatesan S, Ward RJ, Peters TJ. Effect of chronic ethanol feeding on the hepatic secretion of very-low-density lipoproteins. Biochim Biophys Acta. 1988;960(1):61-6.

35. Nanji AA, Dannenberg AJ, Jokelainen K, Bass NM. Alcoholic liver injury in the rat is associated with reduced expression of peroxisome proliferator-alpha (PPARalpha)-regulated genes and is ameliorated by PPARalpha activation. J Pharmacol Exp Ther. 2004;310(1):417-24.
36. Lieber CS. Alcoholic fatty liver: its pathogenesis and mechanism of progression to inflammation and fibrosis. Alcohol. 2004;34(1):9-19.

37. Tessari P, Coracina A, Cosma A, Tiengo A. Hepatic lipid metabolism and non-alcoholic fatty liver disease. Nutr Metab Cardiovasc Dis. 2009;19(4):291-302.

38. Tiniakos DG, Vos MB, Brunt EM. Nonalcoholic fatty liver disease: pathology and pathogenesis. Annu Rev Pathol. 2010:5:145-71.

39. Uslusoy HS, Nak SG, Gulten M, Biyikli Z. Non-alcoholic steatohepatitis with normal aminotransferase values. World J Gastroenterol. 2009;15(15):1863-8.

40. Milstein HJ, Bloomer JR, Klatskin G. Serum bile acids in alcoholic liver disease. Comparison with histological features of the disease. Am J Dig Dis. 1976;21(4):281-5.

41. Trinchet JC, Gerhardt MF, Balkau B, Munz C, Poupon RE. Serum bile acids and cholestasis in alcoholic hepatitis. Relationship with usual liver tests and histological features. J Hepatol. 1994;21(2):235-40.

42. Singh DK, Rastogi A, Sakhuja P, Gondal R, Sarin SK. Comparison of clinical, biochemical and histological features of alcoholic steatohepatitis and non-alcoholic steatohepatitis in Asian Indian patients. Indian J Pathol Microbiol. 2010;53(3):408-13.

43. Samuel VT, Liu ZX, Qu X, Elder BD, Bilz S, Befroy D, et al. Mechanism of hepatic insulin resistance in non-alcoholic fatty liver disease. J Biol Chem. 2004;279(31):32345-53.

44. Frederich RC, Hamann A, Anderson S, Lollmann B, Lowell BB, Flier JS. Leptin levels reflect body lipid content in mice: evidence for diet-induced resistance to leptin action. Nat Med. 1995;1(12):1311-4.

45. Paulino G, Barbier de la Serre C, Knotts TA, Oort PJ, Newman JW, Adams SH, et al. Increased expression of receptors for orexigenic factors in nodose ganglion of diet-induced obese rats. Am J Physiol Endocrinol Metab. 2009; 296(4):E898-903.

46. Rees GM, Miller JA, Casey CA, Tuma DJ. Chronic ethanol administration impairs degradation of formaldehyde-treated albumin by the perfused rat iver. Alcohol Clin Exp Res. 1993;17(6):1309-12.

47. Casey CA, Kragskow SL, Sorrell MF, Tuma DJ. Effect of chronic ethanol administration on total asialoglycoprotein receptor content and intracellular processing of asialoorosomucoid in isolated rat hepatocytes. Biochim Biophys Acta. 1990;1052(1):1-8.

48. Casey CA, Kragskow SL, Sorrell MF, Tuma DJ. Ethanol-induced impairments in receptor-mediated endocytosis of asialoorosomucoid in isolated rat hepatocytes: time course of impairments and recovery after ethanol withdrawal. Alcohol Clin Exp Res. 1989;13(2):258-63.

49. Lieber CS. Cytochrome P-4502E1: its physiological and pathological role. Physiol Rev. 1997;77(2):517-544.

50. Clemens DL, Casey CA, Sorrell MF, Tuma DJ. Ethanol oxidation mediates impaired hepatic receptormediated enocytosis. Alcohol Clin Exp Res. 1998; 22(4):778-779.

51. Clemens DL, Tuma DJ, Casey CA. Cyanamide potentiates the ethanolinduced impairment of receptormediatedendocytosis in a recombinant hepatic cell line expressing alcohol dehydrogenase activity. Int JHepatol. 2012:2012:954157

\section{Submit your next manuscript to BioMed Central and we will help you at every step:}

- We accept pre-submission inquiries

- Our selector tool helps you to find the most relevant journal

- We provide round the clock customer support

- Convenient online submission

- Thorough peer review

- Inclusion in PubMed and all major indexing services

- Maximum visibility for your research

Submit your manuscript at www.biomedcentral.com/submit 